\title{
Diagnostic performance and cutoff value for the clock drawing test, semantic verbal fluency, and the short Boston Naming Test in community-dwelling educated older adults with neurocognitive disorders
}

\author{
Reem Mohamed Sabry ELbedewy ${ }^{* \dagger}$ and Mohamed ELokl ${ }^{\dagger}$
}

\begin{abstract}
Background: Little is known regarding the standardized neuropsychological tests available in Arabic. The aim is to determine the diagnostic performance and the best cutoff value for the clock drawing test (CDT), animal category test of semantic verbal fluency (VF), and the short form of the Boston Naming Test (BNT) in identifying patients with mild and major neurocognitive disorders among community-dwelling educated Egyptian older adults using Arabic versions of those tests. Community-dwelling educated male and female older adults aged 60 years or older. Successive patients were attending the outpatient geriatrics clinic at the Ain Shams University Hospital during a 12month study period from January to December 2016. The study was approved by the ethics committee of the Faculty of Medicine, Ain Shams University, Cairo, Egypt. Informed consent to participate in the study was received from each participant and/or his/her next of kin. Patients and/or their next of kin who declined to participate were excluded, as were those who refused to complete the assessment.

A two-step protocol was followed. Step 1: Participants were divided into three groups according to DSM-V diagnostic criteria for neurocognitive disorders. The assessment of cognitive function included Mini-Mental State Examination (MMSE), Clinical Dementia Rating Scale (CDR), patient's current functional status regarding basic and instrumental activities of daily living by the Blessed Dementia Scale (BDS), and patient detailed history and examination based on protocol five of the CERAD assessment packet.

Step 2: The application of the Arabic versions of ELokl et al. 2001 VF test, the BNT, and the CD by a clinical psychologist blinded to the initial assessment results.

Results: In current study, AUC for CD, VF, and BN are $0.807,0.77$, and 0.753 respectively for mild NCD and 0.884 , 0.877 , and 0.839 respectively for major NCD while cutoff values for CD, VF, and BN are 2, 12, and 14 respectively for mild NCD and 2, 9, and 13 respectively for major NCD.
\end{abstract}

Conclusion: Verbal fluency, clock drawing, and Boston naming showed reasonable diagnostic performance in educated Egyptian elderly and should be considered separately or in combination for the assessment of cognitive function. Further research is warranted.

Keywords: Neuropsychological tests, Verbal fluency, Clock drawing test, Boston Naming Test, Neurocognitive disorder, Arabic CERAD, Egypt, Elderly, Dementia

\footnotetext{
* Correspondence: reem_sabry@med.asu.edu.eg

${ }^{\dagger}$ Reem Mohamed Sabry ELbedewy and Mohamed Elokl contributed equally to this work.

Geriatrics and Gerontology department,Faculty of Medicine, Ain Shams University, Cairo, Egypt
} 


\section{Background}

Although Arabic is one of the most widely used languages worldwide, little is known regarding the standardized neuropsychological tests available in Arabic [1]. One of the most widely used assessment packages in dementia is the Consortium to Establish a Registry for Alzheimer's Disease (CERAD) which was established in 1986. The major standardized instruments developed by CERAD are now used by many Alzheimer's disease research centers in the USA and abroad, by physicians in clinical practice, and in population-based surveys $[2,3]$. They have been translated, in whole or in part, into Arabic, Bulgarian, Chinese, Dutch, Finnish, French, German, Hebrew, Hindi (for a rural, illiterate population), Italian, Japanese, Korean, Norwegian, Polish, Portuguese, Russian, Spanish, and Swedish and into English sign language [4]. The CERAD neuropsychological battery and assessment package has been translated into Arabic, adapted and validated for the Egyptian language and culture [5]. For the purpose of the current study, we used the Arabic versions of the subset of tests from the CERAD battery that were included in this study: the animal category test of semantic verbal fluency (VF) [6], the 15-item short version of the Boston Naming Test (BNT) [7], the Mini-Mental State Examination (MMSE) [8], and the clock drawing test (CDT) [9]. All have been translated into Arabic language and have been validated as a part of the Arabic version [5]. The animal category test of semantic VF is a measure of impairment in verbal production and a test of semantic memory and language. It is rapidly administered, readily available, and easy to administer and interpret. The individual is asked to name all the animals that he/she can name within $60 \mathrm{~s}$. This task can be understood by patients with cognitive impairment and is sensitive to Alzheimer's disease [10]. The BNT is a test of language ability in which participants are asked to name objects depicted by line drawings [7]. The short BNT contains five high-frequency, five medium-frequency, and five low-frequency items. Each participant is presented with 15 line drawings in an accompanying flipbook. The correct names for the objects are listed on the Response Sheet of CERAD Form J2. The third test included was the CDT. It was originally used to assess visuoconstructive abilities. It has been proposed as a quick screening test for cognitive dysfunction [11]. The strength and weakness of the CDT lies in the number of cognitive, motor, and perceptual functions that must be used simultaneously to successfully complete the task, which requires visual memory, visuospatial abilities, orientation, conceptualization of time, executive function, auditory comprehension, motor programming, numerical knowledge, semantic instruction, inhibition of distracting stimuli, concentration, and frustration tolerance [12]. Serial CDTs can be used to follow a patient with progressive dementia or to observe recovery from delirium [13]. The MMSE is in some ways the best known and most widely used measure of cognition in clinical practice worldwide [14], and it represents a benchmark against which all newer tools can be measured [15]. It is an easily administered, simple, and widely available test that assesses orientation, memory, attention and calculation, language, and visual construction. The MMSE takes approximately $10 \mathrm{~min}$ to administer, and its total score is 30 . It has been translated and adapted for the culture of elderly Arabic-speaking Egyptians as a part of the Arabic translation and adaptation of the CERAD battery [5].

\section{Aim}

The aim is to determine the diagnostic performance and the best cutoff value for the clock drawing test (CDT), animal category test of semantic verbal fluency (VF), and the short form of the Boston Naming Test (BNT) in identifying patients with mild and major neurocognitive disorders among community-dwelling educated Egyptian older adults using Arabic versions of those tests.

\section{Methods \\ Participants}

Community-dwelling educated male and female older adults aged 60 years or older. Education is defined as 6 years or more of formal education. The setting, sample size, and selection criteria are as follows: all patients attending the outpatient geriatric clinic at Ain Shams University Hospital, Cairo, Egypt, in the period from January 1, 2016, till December 31, 2016, excluding, at the start of the study, those patients who or their next of kin refused to participate in the study. (Successive patients attending the outpatient geriatric clinic at Ain Shams University Hospital during a 12-month study period from January to December 2016. Patients were excluded if they or their next of kin declined to participate.) We estimated the minimum sample size required, based on the prevalence of cognitive disorders and both sensitivity and specificity of the screening and diagnostic values of tests studied. The power is set to be at least $80 \%$ and the $p$ value and is set to be less than 0.05 . The calculated sample size is found to be 110 with minimal number of 35 in each studied group.

A two-step protocol was followed.

Step 1: Participants were divided into three groups according to DSM-V diagnostic criteria for neurocognitive disorders. The assessment of cognitive function included Mini-Mental State Examination (MMSE), Clinical Dementia Rating Scale (CDR), patient's current functional status regarding basic and instrumental activities of daily living by the Blessed Dementia Scale (BDS) [16], and patient detailed history and examination based on protocol 
five of the CERAD assessment packet. Those with MMSE score of less than 24 were identified as having cognitive impairment $[5,17]$ : CDR score, $0=$ no dementia; $0.5=$ uncertain or deferred diagnosis; $1=$ mild dementia; 2 = moderate dementia; 3 = severe dementia; 4 $=$ profound dementia; $5=$ terminal dementia.

Blessed Dementia Scale: The original Dementia Scale of Blessed et al. has been modified by minor wording changes and by the deletion of questions related to personality, interests, and drives. The informant is asked to rate, on a 3- or 4-point scale as indicated, the subject's cognitive ability during the preceding 6 months, as compared with premorbid ability in performing practical tasks of everyday living. Fully preserved capacity is represented by a total score of 0 , whereas maximum impairment is reflected by a total score of 17 . Less than four is cognitively unimpaired, 4-9 is mild impairment, and more than nine is moderate to severe impairment.

Step 2: The application of the Arabic versions of EL OKL et al. 2001 VF test, the BNT, and the CD by a clinical psychologist blinded to the initial assessment results.

\section{Statistical method}

The collected data was revised, coded, tabulated, and entered into a PC using the Statistical Package for the Social Sciences (IBM SPSS 20.0). The analyses performed were determined by the type of data obtained for each parameter. The sensitivity and specificity of each test are calculated, and the receiver operating characteristic (ROC) curves were drawn. The area under the curve
(AUC) is a measure of diagnostic accuracy. Interpretation of areas under the curve (AUCs) is as follows: $0.90-1=$ excellent, $0.80-0.90=$ good, $0.70-0.80=$ fair, $0.60-0.70=$ poor, $0.50-0.60=$ fail. The cutoff values were determined as the values with the highest balanced sensitivity and specificity.

\section{Results}

The study sample is 122 community-dwelling educated older adults who attended the outpatient geriatric department clinic at the Ain Shams University Hospitals. $32.79 \%$ of the samples (40 subjects) have mild neurocognitive disorder while $38.52 \%$ (47 subjects) have major neurocognitive disorder. As regards the mean age $\pm S D$, it is $70.1 \pm 6.73$ for the group with mild neurocognitive disorder (NCD) and 71.1 \pm 6.24 for the group with major neurocognitive disorder. As regards gender, males are $62(50.8 \%)$ of the sample, $45.2 \%$ of major NCD, and $24.2 \%$ of mild NCD.

Figure 1 and Table 1 show the statistical findings for the mild neurocognitive disorder group. AUC for CD, $\mathrm{VF}$, and $\mathrm{BN}$ are $0.807,0.77$, and 0.753 respectively while the cutoff values for $\mathrm{CD}, \mathrm{VF}$, and $\mathrm{BN}$ are 2,12 , and 14 respectively.

Figure 2 and Table 2 show the statistical findings for the major neurocognitive disorder group.

AUC for CD, VF, and $\mathrm{BN}$ are 0.884, 0.877, and 0.839 respectively while the cutoff values for $\mathrm{CD}, \mathrm{VF}$, and $\mathrm{BN}$ are 2,9 , and 13 respectively. So, the three tests are good tests for major NCD.

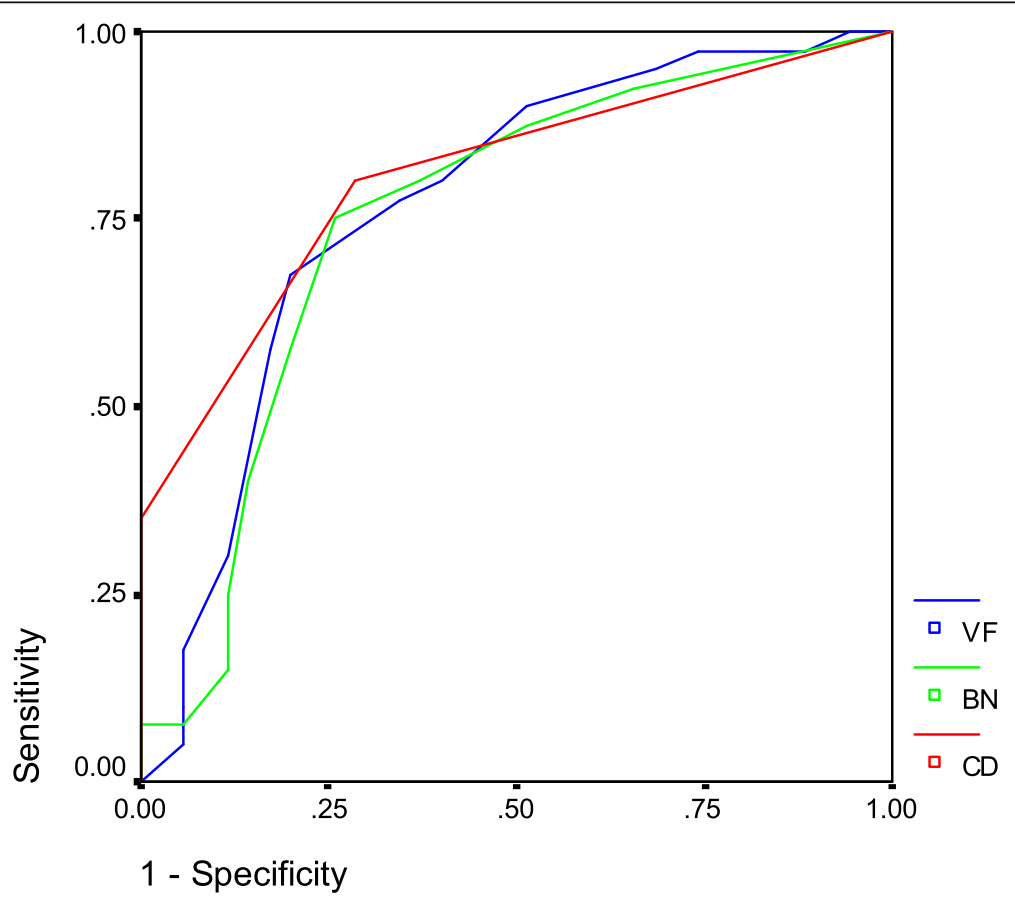

Fig. 1 ROC for $V F, B N$, and $C D$ tests in the mild neurocognitive disorder group 
Table 1 Diagnostic performance and cutoff values for CD, VF, and BN in the mild neurocognitive disorder group

\begin{tabular}{|c|c|c|c|c|c|c|c|}
\hline & \multirow[t]{2}{*}{ AUC } & \multirow{2}{*}{$\begin{array}{l}\text { St. } \\
\text { error }\end{array}$} & \multicolumn{2}{|c|}{ Confidence interval } & \multirow[t]{2}{*}{ Cutoff } & \multirow[t]{2}{*}{ Sensitivity } & \multirow[t]{2}{*}{ Specificity } \\
\hline & & & Lower bound & Upper bound & & & \\
\hline$C D$ & .807 & .050 & .709 & .906 & 2 & .800 & .286 \\
\hline VF & .770 & .057 & .659 & .881 & 12 & .970 & .887 \\
\hline $\mathrm{BN}$ & .753 & .059 & .637 & .868 & 14 & .925 & .657 \\
\hline
\end{tabular}

\section{Discussion}

With the projected increase in the elderly population and the expected rise in the prevalence of dementia, early case identification is necessary for planning and delivering clinical services. The effectiveness of dementia screening depends on the availability of suitable screening tools with good sensitivity and specificity to confidently distinguish normal cognitive function from minor and major NCD. There is a demand for rapidly administered, sensitive, and reliable Arabic versions of cognitive assessments particularly those which are designed for identifying older adults with minor and major neurocognitive impairment. To meet this need, we used in the current study the Arabic version of CDT, VF animal category, and BNT as a part of the Arabic version of CERAD neuropsychological battery. The CERAD battery was translated into the Arabic language and validated in the Egyptian elderly. We chose rapidly administered and sensitive tools to find out the best cutoff for patients with minor and major neurocognitive disorders in educated Egyptian older adults. Our results present the best cutoff values that discriminate between cognitively healthy individuals and those with minor and major NCD. Our study included 122 community-dwelling educated older adults matched for age and gender, attending outpatient clinics at one of the largest tertiary hospitals in Cairo, Egypt. $71.3 \%$ of participants (number $=87$ ) have either mild or major NCD. This could be explained by the fact that the participants were attending the outpatient clinics of the tertiary hospital with different comorbidities. This was not a community-based study, although the participants were community-dwelling and not living in residential care or nursing homes. Our study population represents the community-dwelling educated elderly adults who attended the outpatient general geriatric clinic with different medical illnesses. To our knowledge according to available resources to us, there are no available previous studies with the same aim and tools in Egypt to compare our results with, but there are studies for VF, CDT, and BN performance and cutoff points for other purposes. In the current study results, AUC for $\mathrm{CD}, \mathrm{VF}$, and $\mathrm{BN}$ for participants with minor NCD are $0.807,0.77$, and 0.753 respectively. It means that $\mathrm{CD}$ is a good test while VF and $\mathrm{BN}$ are fair tests for detection of minor NCD. For participants with major $\mathrm{NCD}, \mathrm{AUC}$ for $\mathrm{CD}, \mathrm{VF}$, and $\mathrm{BN}$ are 0.884, 0.877, and 0.839

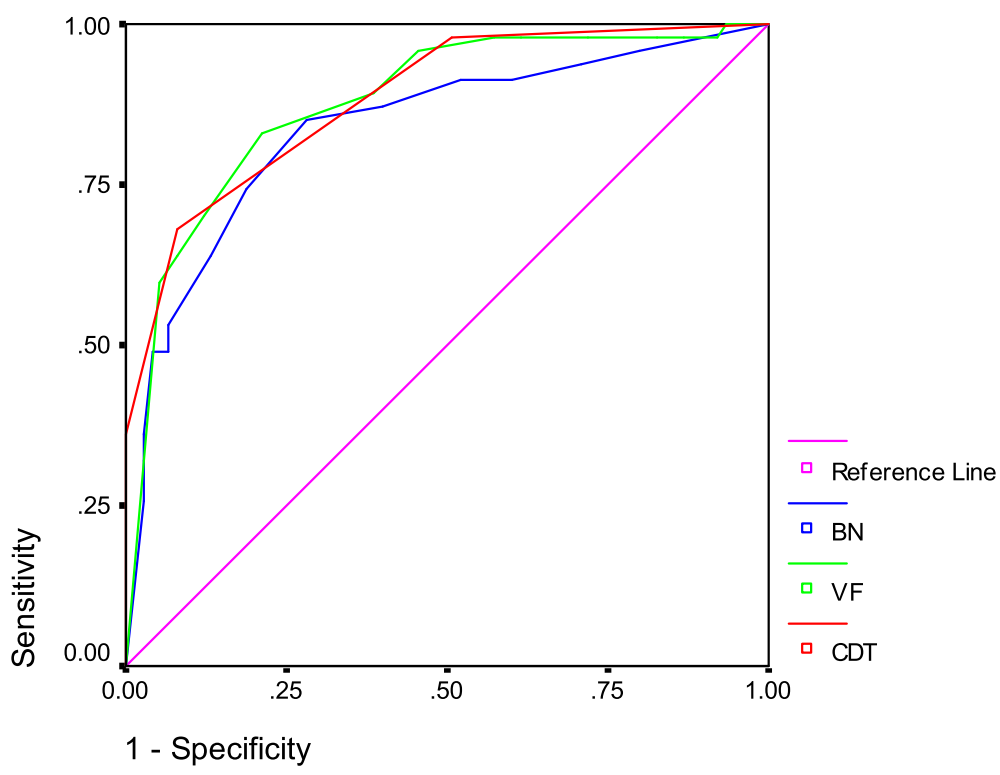

Fig. 2 ROC for $V F, B N$, and $C D$ tests in the major neurocognitive disorder group 
Table 2 Diagnostic performance and cutoff values for CD, VF, and BN in major neurocognitive disorder group

\begin{tabular}{|c|c|c|c|c|c|c|c|}
\hline \multirow{2}{*}{\multicolumn{2}{|c|}{$A \cup C$}} & \multirow{2}{*}{$\begin{array}{l}\text { St. } \\
\text { error }\end{array}$} & \multicolumn{2}{|c|}{ Confidence interval } & \multirow[t]{2}{*}{ Cutoff } & \multirow[t]{2}{*}{ Sensitivity } & \multirow[t]{2}{*}{ Specificity } \\
\hline & & & Lower bound & Upper bound & & & \\
\hline$\overline{C D}$ & .884 & .031 & .824 & .944 & 2 & .979 & .507 \\
\hline VF & .877 & .033 & .813 & .941 & 9 & .979 & .827 \\
\hline $\mathrm{BN}$ & .839 & .039 & .762 & .916 & 13 & .957 & .800 \\
\hline
\end{tabular}

respectively. It means that the three tests are good tests for the detection of major NCD. In addition, the cutoff values for $\mathrm{CD}, \mathrm{VF}$, and $\mathrm{BN}$ are 2,12 , and 14 respectively for mild NCD and 2, 9, and 13 respectively for major NCD. According to Wiechmann et al. [18] study, ROC analysis indicated that the CDT was able to distinguish between normal elders and those with a dementia diagnosis. The cutoff score for differentiating patients with Alzheimer's disease from normal participants was $\leq 3$. The cutoff score for differentiating those with vascular disease from normal participants was $\leq 3$. Mirandez [19] found that the cutoff score for the animal category test of VF was 14 , and the AUC $=0.794$. In comparison, Brucki et al. [20] found that the cutoff points were 9 for people with less than 8 years of education and had a mean of 13 for those with higher educational levels. With the abovementioned ROC and best cutoff values in our study, it is clear that our results are comparable to those studies, and those tests can be used to screen for cognitive impairment in educated Egyptian older adults.

\section{Conclusion}

The study presents the best cutoff for a group of neuropsychological tests which will help to advance research in the area of cognitive impairment in Egypt. Verbal fluency, clock drawing, and Boston naming showed reasonable diagnostic performance in educated Egyptian elderly and should be considered separately or in combination for the assessment of cognitive function.

\section{Recommendations}

Further research on a larger number and on a more diverse group of the population is warranted.

\section{Abbreviations}

AUC: The area under the curve; BDS: Blessed Dementia Scale; BNT: Boston Naming Test; CDT: Clock drawing test; CERAD: The Consortium to Establish a Registry for Alzheimer's Disease; MMSE: Mini Mental State Examination; NCD: Neurocognitive disorder; ROC: Receiver operating characteristic; SD: Standard deviation; SPSS: Statistical Package for the Social Sciences; VF: Verbal fluency

\section{Acknowledgements}

Not applicable.

\section{Authors' contributions}

Both authors, RE and ME, equally participated in the conception, design of the work, data collection, interpretation of the data, review of the results' analysis, writing, revising, and editing this manuscript. Both authors read and approved the final submitted manuscript.

\section{Authors' information}

Both authors are associate professors at the Geriatrics and Gerontology Department, Faculty of Medicine, Ain Shams University, Cairo, Egypt. Dr. Mohammed ELOKL passed MRCP UK on November 2007, and currently, he is working as a Consultant of Geriatric Medicine in Weston Area NHS Trust in England, UK.

\section{Funding}

Not applicable.

\section{Availability of data and materials}

The data generated and analyzed during this study are included in this article and available from the corresponding author on further reasonable request.

\section{Ethics approval and consent to participate}

The study was approved by the ethics committee of the Faculty of Medicine, Ain Shams University, Cairo, Egypt. Reference number is not available. Informed oral consent to participate in the study was received from each participant and/or his/her next of kin. Patients and/or their next of kin who declined to participate were excluded, as were those who refused to complete the assessment.

\section{Consent for publication}

Not applicable.

\section{Competing interests}

The authors declare that they have no competing interests.

Received: 4 December 2019 Accepted: 24 December 2019

Published online: 03 March 2020

\section{References}

1. Fasfous AF, Al-Joudi HF, Puente AE, Pérez-García M (2017) Neuropsychological measures in the Arab World: a Systematic Review. Neuropsychol Rev 27(2):158-173

2. Fillenbaum GG, Heyman A, Huber MS, Ganguli M, Unverzagt FW (2001) Performance of elderly African American and White community residents on the CERAD Neuropsychological Battery. J Int Neuropsych Soc 7:502-509

3. Morris JC, Heyman A, Mohs RC et al (1989) The Consortium to Establish a Registry for Alzheimer's Disease (CERAD). Part I: Clinical and neuropsychological assessment of Alzheimer's disease. Neurology 39:11591165

4. CERAD - Consortium to Establish a Registry for Alzheimer's disease (2018) Duke center for the study of aging and human development. https://sites. duke.edu/centerforaging/cerad. Accessed 28/07/2018.

5. Elokl M/Elbanouby M/Mortagy M/elAtrebi M/ELsabwa M (2001) Ain shams university, faculty of medicine department of Geriatrics. Prevalence of AD, and other types of dementia in Egypt. Int Psychogeriatric, 13, Supplement 2.

6. Benton AL (1968) Differential behavioral effects in frontal lobe disease. Neuropsychologia 6:53-60. https://doi.org/10.1016/0028-3932(68)90038-9

7. Kaplan EF, Goodglass H, Weintraub S (1978) The Boston Naming Test. Veterans Administration Medical Center, Boston

8. Folstein et al (1975) Mini-mental state. "A practical method for grading the cognitive state of patients for the clinician". J Psychiatr Res 12:189-198

9. Freedman MI, Leach L, Kaplan E, Winocur G, Shulman KJ, Delis DC (eds) (1994) Clock drawing. Oxford University Press, Oxford

10. Budson AE, Solomon PR (2016) Memory loss, Alzheimer's disease, and dementia (second edition) Chapter 2- Evaluating the patient with memory loss or dementia, pp 5-38 https://doi.org/10.1016/B978-0-323-28661-9. 00002-0 
11. Cucinotta D, Reggiani A, Galletti L, Rasciti L, De Notariis S (2004) Preventivecomprehensive assessment (PCA): a new screening method for subclinical cognitive problems. Arch Gerontol Geriatr Suppl 9:97-102

12. Shulman Kl, Shedletsky R, Silver IL (1986) The challenge of time: clock-drawing and cognitive function in the elderly. Int I Geriatr Psychiatr 1:135-140

13. Shulman Kl (2000) Clock-drawing: is it the ideal cognitive screening test? Int J Geriatric Psychiatr 15:548-561

14. Sheehan B (2012) Assessment scales in dementia. Ther Adv Neurol Disord 5(6):349-358. https://doi.org/10.1177/1756285612455733

15. Larner AJ (2017) Cognitive screening instruments: a practical approach (2nd edition). Springer, London, $\mathrm{P} 38$

16. Blessed G, Tomlinson BE, Roth M (1968) The association between quantitative measures of dementia and senile change in the cerebral gray matter of elderly subjects. Br J Psychiatry. 114:797-811

17. Heyman A, Fillenbaum GG, Mirra SS (1990) Consortium to Establish a Registry for Alzheimer's Disease (CERAD): clinical, neuropsychological, and neuropathological components. Aging (Milano) 2(4):415-424

18. Wiechmann AR, Hall JR, O'Bryant S (2010) The four-point scoring system for the clock drawing test does not differentiate between Alzheimer's disease, and vascular dementia. Psychol Rep. 106(3):941-948

19. Mirandez RM, Aprahamian I, Talib LL, Forlenza OV, Radanovic M (2017) Multiple category verbal fluency in mild cognitive impairment and correlation with CSF biomarkers for Alzheimer's disease. Int Psychogeriatr 29(6):949-958. https://doi.org/10.1017/S1041610217000102 Epub 2017 Feb 9

20. Brucki SM, Malheiros SM, Okamoto IH, Bertolucci PH (1997) Normative data on the verbal fluency test in the animal category in our milieu. Arq Neuropsiquiatr. 55(1):56-61

\section{Publisher's Note}

Springer Nature remains neutral with regard to jurisdictional claims in published maps and institutional affiliations.

\section{Submit your manuscript to a SpringerOpen ${ }^{\circ}$ journal and benefit from:}

- Convenient online submission

- Rigorous peer review

- Open access: articles freely available online

- High visibility within the field

- Retaining the copyright to your article

Submit your next manuscript at $\boldsymbol{\nabla}$ springeropen.com 